\title{
CONVOLUTIONAL NEURAL NETWORK ARCHITECTURES PERFORMANCE EVALUATION FOR FISH SPECIES CLASSIFICATION
}

\author{
HAMIZAH ISMAIL ${ }^{1 *}$,AHMAD FAISAL MOHAMAD AYOB ${ }^{1}$,AIDY @, MUHAMED SHAWAL \\ M MUSLIM $^{2}$ AND MOHAMAD FAKHRATUL RIDWAN ZULKIFLI ${ }^{1}$
}

${ }^{1}$ Faculty of Ocean Engineering and Informatics, ${ }^{2}$ Institute of Oceanography and Environment, Universiti Malaysia Terengganu, Kuala Nerus, 21030 Terengganu, Malaysia.

*Corresponding author: hamizahismail929@gmail.com

Submitted final draft: 11 August $2020 \quad$ Accepted: 11 September $2020 \quad$ http://doi.org/10.46754/jssm.2021.07.010

\begin{abstract}
Fish image classification tool is important in the field of ichthyology. In this paper, we present a fish image classification benchmark comparison across different types of convolutional neural network $(\mathrm{CNN})$. CNN extracts features from labeled image data to solve classification problems. CNN models were trained to classify fish images using transfer learning with data augmentation. CNN models consisting of AlexNet, GoogLeNet, and ResNet were incorporated in the benchmark tests. A dataset of 18,000 fish images across 18 categores, were split into 5,400 images for validation (30\%) and 12,600 images for training (70\%) dataset. Such neural network models show high accuracy up to $99.85 \%$ (AlexNet), 96.39\% (GoogLeNet) and 99.51\% (ResNet-50). To evaluate the performance of each framework, the analysis presented consists of classification accuracy, learning curve, validation test, top-five prediction and confusion matrix. The work presented here has shown its potential to contribute towards accurate development of state-of-the-art fish classification tools. It is envisioned that these $\mathrm{CNN}$ algorithms have the potential to assist in fish image classification problems with high accuracy despite visually similar features of images in the dataset.
\end{abstract}

Keywords: Fish image classification, marine technology, artificial intelligence, deep learning.

\section{Introduction}

The development of automated

fish classification tools is the most recent trend in machine learning (Man, 2016 \& Sarigül et al., 2017). Fish classification tasks are beneficial to aid individuals without taxonomic backgrounds, such as data collectors and entry-level ichthyologists. Fish image classifications were explored using machine learning algorithms. Serna and Segura (2014), designed an algorithm and defined a range of new features that use pattern recognition with artificial neural networks (ANN) for Europe and South America fish species identifications with $91.65 \%$ accuracy. Deep artificial neural networks with a different number of layers and various filter sizes are used for comparison of different deep structures for fish classification tasks in Queensland University of Technology fish dataset (Sarigül \& Avci, 2017).
Recently, Convolutional Neural Networks (CNNs) have shown a remarkable accuracy for image classification (Krizhevsky et al., 2012; Russakovsky et al., 2015). Deep learning extracts features from the image dataset with different layered architectures consisting of convolutional layers, pooling layers, and finally, connected to a fully connected layer to produce the output. Currently, CNN has been studied by many researchers and applied to a wide range of classification problems. There are some works that applied CNNs in numerous studies, as shown in Table 1. In fish classification, the use of CNN is challenging due to the visually similar features of fish. The CNN is proposed to provide high accuracy in fish classification as it learns the boundary and color features of the fish images. Table 2 shows the fish image classification approach using CNN. 
Table 1: Summary of related deep learning works for other types of image classification

\begin{tabular}{|c|c|c|c|c|}
\hline References & Categories & Data & Architecture & Results \\
\hline (Liu, 2020) & $\begin{array}{l}\text { Soft-shell shrimp } \\
\text { and sound shrimp }\end{array}$ & $\begin{array}{l}\text { Images taken using } \\
\text { CCD camera }\end{array}$ & Deep-ShrimpNets-12 & $\begin{array}{l}\text { Mean accuracy } \\
\text { precision of } \\
97.20 \%\end{array}$ \\
\hline $\begin{array}{l}\text { (Thenmozhi et } \\
\text { al., 2019) }\end{array}$ & $\begin{array}{l}40,24 \text { and } 40 \\
\text { insect classes }\end{array}$ & $\begin{array}{l}\text { NBAIR, Xie1 and } \\
\text { Xie2 insect dataset }\end{array}$ & $\begin{array}{l}\text { CNN with } 6 \\
\text { convolution layers and } \\
5 \text { pooling layers }\end{array}$ & $\begin{array}{l}\text { Accuracy of } \\
96.75 \% \text { (NBAIR), } \\
97.47 \%(\mathrm{Xie} 1) \\
\text { and } 95.97 \% \\
\text { (Xie } 2)\end{array}$ \\
\hline $\begin{array}{l}\text { (Steinbrener } e t \\
\text { al., 2019) }\end{array}$ & $\begin{array}{l}13 \text { classes of fruit } \\
\text { and vegetable }\end{array}$ & $\begin{array}{l}\text { Images taken using a } \\
\text { hyperspectral camera }\end{array}$ & $\begin{array}{l}\text { Convolutional layer } \\
\text { with } 3 \text { kernels of } \\
\text { size }(3 \times 3 \times 5) \text { and } \\
\text { rectified linear unit as } \\
\text { activation }\end{array}$ & $\begin{array}{l}\text { Accuracy of } \\
92.23 \%\end{array}$ \\
\hline (Ríos et al., 2019) & $\begin{array}{l}14 \text { classes of } \\
\text { coral species }\end{array}$ & $\begin{array}{l}\text { RSMAS small coral } \\
\text { texture dataset }\end{array}$ & $\begin{array}{l}\text { ResNet-152 with } \\
\text { data augmentation } \\
\text { (zooming) }\end{array}$ & $\begin{array}{l}\text { Accuracy of } \\
98.63 \%\end{array}$ \\
\hline $\begin{array}{l}\text { (Kozłowski et al., } \\
\text { 2019) }\end{array}$ & 6 varieties & $\begin{array}{l}\text { Scanned images of } \\
\text { barley }\end{array}$ & $\begin{array}{l}\text { Convolutional layers } \\
\text { with } 64 \text { filters of size } \\
3 \times 3\end{array}$ & $\begin{array}{l}\text { Accuracy of } \\
93.21 \%\end{array}$ \\
\hline (Xu \& Sun, 2018) & $\begin{array}{l}\text { Normal flesh and } \\
\text { gaping categories }\end{array}$ & $\begin{array}{l}\text { Salmon muscle } \\
\text { gaping images taken } \\
\text { from digital camera }\end{array}$ & AlexNet & $\begin{array}{l}\text { Correct } \\
\text { classification rates } \\
\text { (CCRs) of } 91.60 \% \\
\text { for test data set }\end{array}$ \\
\hline $\begin{array}{l}\text { (Wang et al., } \\
\text { 2018) }\end{array}$ & 6 types of plants & $\begin{array}{l}\text { Original images } \\
\text { of crop and weed } \\
\text { captured by UAV }\end{array}$ & $\mathrm{CNN}$ with 5 layers & $\begin{array}{l}\text { Accuracy } 95.60 \% \\
\text { for } 300 \times 300 \text { input } \\
\text { image resolutions }\end{array}$ \\
\hline $\begin{array}{l}\text { (Tyagi et al., } \\
\text { 2018) }\end{array}$ & $\begin{array}{l}11 \text { classes of } \\
\text { phenotypical } \\
\text { deformation }\end{array}$ & $\begin{array}{l}\text { Phenotypes zebrafish } \\
\text { embryo obtained } \\
\text { using high-resolution } \\
\text { imaging }\end{array}$ & Fine-Tuned VGG16 & Accuracy $83.55 \%$ \\
\hline $\begin{array}{l}\text { (Verma \& Gupta, } \\
\text { 2018) }\end{array}$ & $\begin{array}{l}20 \text { species of } \\
\text { wild animal }\end{array}$ & $\begin{array}{l}\text { Images taken using } \\
\text { camera trap }\end{array}$ & Deep CNN & $\begin{array}{l}\text { Accuracy of } \\
91.40 \%\end{array}$ \\
\hline $\begin{array}{l}\text { (Dangelo \& } \\
\text { Tennefoss, 2015) }\end{array}$ & $\begin{array}{l}121 \text { classes of } \\
\text { plankton }\end{array}$ & $\begin{array}{l}\text { Gray-scale images } \\
\text { of photo plankton } \\
\text { species }\end{array}$ & Network in Network & $\begin{array}{l}26.10 \% \text { correct } \\
\text { class prediction }\end{array}$ \\
\hline
\end{tabular}

For pre-trained $\mathrm{CNN}$ models in this study, AlexNet, GoogLeNet, and Residual Net (ResNet) were utilized. The AlexNet architecture stacked multiple convolutional layers that allow for more complex features of the input vector to be selected (Krizhevsky et al., 2012). It contains eight learned layers with weights made up of five convolutional layers, max-pooling layers, dropout layers, and three fully connected layers. AlexNet used a novel activation function,
Rectified Linear Unit (ReLU) that reduces its training time. ReLU is applied to the output of every convolutional layer and fully connected layer. Local Response Normalization (LRN) implements competition between neurons at the same location but in different feature maps. LRN follows the first and second convolutional layers (Krähenbühl, et al., 2015). Data augmentation and dropout layer were also implemented in the network to prevent overfitting of data. 
Table 2: Summary of related works using deep learning for fish image classification

\begin{tabular}{|c|c|c|c|c|}
\hline References & Categories & Data & Architecture & Results \\
\hline (Liawatimena et al., 2019) & 3 species & Fish images & AlexNet & $\begin{array}{l}\text { Accuracy of } \\
99.63 \%\end{array}$ \\
\hline (Kratzert et al., 2018) & 15 species & $\begin{array}{l}\text { Images captured from } \\
\text { videos }\end{array}$ & VGG-16 & $\begin{array}{l}\text { Accuracy of } \\
93.00 \%\end{array}$ \\
\hline (Rathi, Jain, \& Indu, 2018) & 23 species & $\begin{array}{l}\text { Images captured from } \\
\text { videos }\end{array}$ & $\mathrm{CNN}$ & $\begin{array}{l}\text { Accuracy of } \\
96.29 \%\end{array}$ \\
\hline (Siddiqui et al., 2018) & 16 species & $\begin{array}{l}\text { Images captured from } \\
\text { videos }\end{array}$ & $\mathrm{CNN}$ & $\begin{array}{l}\text { Accuracy of } \\
94.30 \%\end{array}$ \\
\hline (Polzounov et al., 2016) & 1 species & Right whale images & $\mathrm{CNN}$ & $\begin{array}{l}\text { Accuracy of } \\
99.18 \%\end{array}$ \\
\hline (Salman et al., 2016) & 15 species & $\begin{array}{l}\text { Images captured from } \\
\text { videos }\end{array}$ & $\mathrm{CNN}$ & $\begin{array}{l}\text { Accuracy of } \\
90.00 \%\end{array}$ \\
\hline (Choi, 2015) & 15 species & $\begin{array}{l}\text { Images captured from } \\
\text { videos }\end{array}$ & GoogLeNet & $\begin{array}{l}\text { Precision of } \\
81.00 \%\end{array}$ \\
\hline
\end{tabular}

Moreover, the GoogLeNet has 22 layers of deep network with 9 inception units and a fully connected layer before the output. Inception units or modules are inspired by inception layers that have variable receptive fields that are created by different kernel sizes (Alom et al., 2018). The architecture uses a combination of inception modules, each including pooling, convolutions at different scales and filter concatenation operations. The inception module has two layers and 6 convolutional blocks that are an essential component of GoogLeNet. It uses the parallel combination of $1 \times 1,3 \times 3$ and $5 \times 5$ filters; in GoogLeNet, the 1x1 filter works as a feature selector that reduces the number of parameters, which reduces the processing speed (Szegedy et al., 2015).

In addition, the ResNet introduces a principled approach to add shortcut connections for every two layers to a VGG style-network. ResNet is developed with many different numbers of layers such as 34, 50, 101, and 152. ResNet-50 is the most popular network consisting of 50 layer residual networks that have 50 layers with 49 convolutional layers and a final fully connected classification layer. The ResNet network consists of several basic residual blocks; ReLU activation functions and convolution layers. The pre-trained $\mathrm{CNN}$ and its properties are presented in Table 3.

In this study, we used the $\mathrm{CNN}$ architectures with different depths and structures to determine performance evaluation for fish image classification using transfer learning. Transfer learning was evident to be a remarkable and effective way to train a large network using insufficient training data without overfitting (Huang, Pan, \& Lei, 2017). The different CNN architectures are investigated until the latest well-known architectures published in 2017; AlexNet, GoogLeNet, and ResNet-50. The transfer learning properties of Convolutional

Table 3: Pre-trained CNN models with its properties

\begin{tabular}{lccc}
\hline \multicolumn{1}{c}{ References } & $\begin{array}{c}\text { Architecture } \\
\text { Layers }\end{array}$ & $\begin{array}{c}\text { Parameters } \\
\text { (Millions) }\end{array}$ & $\begin{array}{c}\text { Image Input Size } \\
\text { (pixels) }\end{array}$ \\
\hline AlexNet (Krizhevsky et al., 2012) & 8 & 61.0 & $227 \times 227$ \\
GoogLeNet (Szegedy et al., 2015) & 22 & 7.0 & $224 \times 224$ \\
ResNet-50 (Wu et al., 2017) & 50 & 25.6 & $224 \times 224$ \\
\hline
\end{tabular}


Neural Networks are exploited for fish image classification. Then, three different $\mathrm{CNN}$ architectures (AlexNet, GoogLeNet, and ResNet-50) are trained and evaluated on fish species images dataset, namely FishNet. The performances of pre-trained $\mathrm{CNN}$ models for fish species classification are compared specifically, using a new test image.

\section{Materials and Methods}

\section{Data Collection}

FishNet dataset was collected through Google search engine and QUT fish dataset (Anantharajah et al., 2014). It consists of realworld images of fish captured in conditions defined as controlled, out-of-the-water and insitu. In our experiments, a total of 736 images were pre-processed via data augmentation method, which resulted in 29,275 fish images (Table 4). For each fish species, there are between 1,000 and 2,800 of images. However, in network training, the lowest number, which is 1000 images in each category, is automatically selected to avoid bias. Thus, a total of 18,000 fish images were used for this experiment. Such a number of images were divided randomly (70\%-30\%) into training images $(12,600)$ images and test images $(5,400)$ set, respectively. The original size of each image is not more than $600 \times 600$ pixels, with random dimensions of images. Then, the collection of data images was down-sampled to the same dimensions as the images used for the pre-trained network, i.e. $227 \times 227$ pixels for AlexNet and 224x224 pixels for GoogLeNet, and ResNet-50 respectively.

Table 4: Fish sample images dataset of different species.

\begin{tabular}{|c|c|c|}
\hline Species & Sample Images & Total Images \\
\hline Atule mate & & 1760 \\
\hline Caranx ruber & & 1950 \\
\hline Cetoscarus bicolor & & 1640 \\
\hline Decapterus russelli & & 1000 \\
\hline Epinephelus areolatus & & 1404 \\
\hline Epinephelus fasciatus & & 2800 \\
\hline
\end{tabular}


Halichoeres argus



1800

Hemiglyphidodon plagiometopon

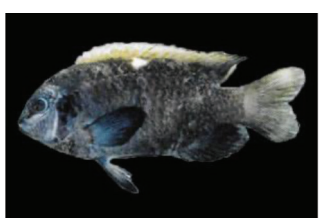

1000

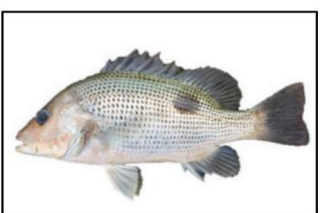

Lutjanus inermis

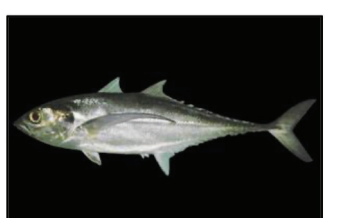

1000

Megalaspis cordyla



Monodactylus argenteus

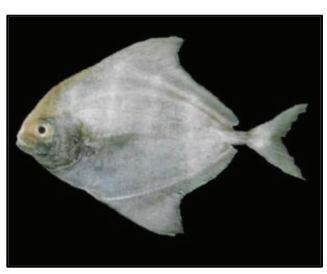

Parastromateus niger

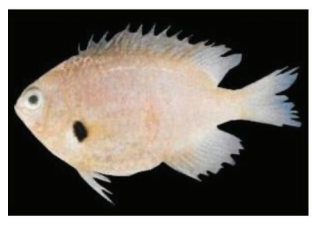

Pomacentrus alexanderae

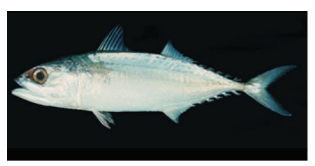

Rastrelliger kanagurta

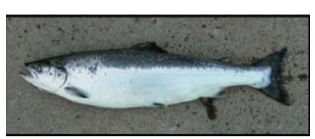

1560

Salmo salar 
Scomber scombus



1600

Scomberoides commersonnianus



1080

Scomberoides tala



1320

\section{Data Pre-processing}

Data augmentation is an image pre-processing step before serving as data input to the network. This step is very important to prevent the overfitting of the network, hence increasing the robustness of the $\mathrm{CNN}$ models. As explained in the previous section, 736 images were augmented to enrich the dataset size in terms of volume and orientation. The training images were augmented by 36 rotations; in $10^{\circ}$ rotation increments. Figure 1 shows an example of data augmentation, where; (a) is the original image, (b)-(j) are the rotated images. After the augmentation, 29,275 images were generated.



(a) Original image

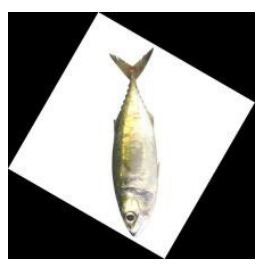

(f) 50-degree rotation

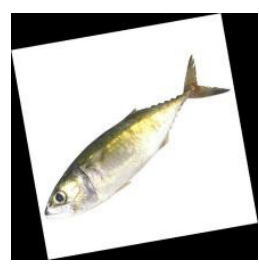

(b) 10-degree rotation

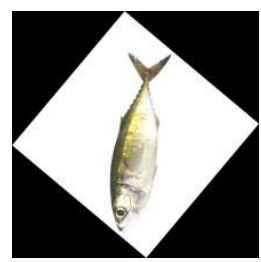

(g) 60-degree rotation

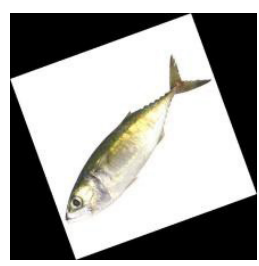

(c) 20-degree rotation



(h) 70-degree rotation

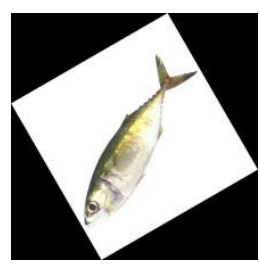

(d) 30-degree rotation

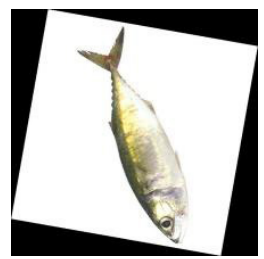

(i) 80-degree rotation

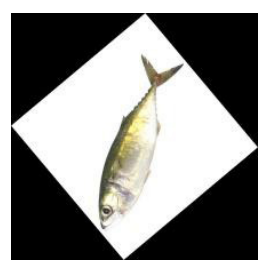

(e) 40-degree rotation



(j) 90-degree rotation

Figure 1: Data augmentation examples 


\section{Case Study}

Transfer learning is an approach that takes layers from a network trained on a large dataset, transfer weights and layers from pre-trained models then replace the final classification layer and finetune on the image classification task. Initial pre-trained networks are trained on a subset of ImageNet database, on more than a million images to classify images into 1,000 categories. In this case study, we compare the performance evaluations of different CNNs using transfer learning method to classify images into 18 fish species. Illustrated in Figure 2 is the framework of a pre-trained CNN case study for fish image classification using transfer learning.

The dataset of labeled fish species images is resized with respect to the pre-trained image size requirement following the pre-trained model properties in Table 3 . The input image size is denoted as the spatial dimensional, (width and height) times the number of 3 of colour components; red, green and blue. Next, the pretrained CNNs are chosen. Three final layers of the pre-trained network (fully connected layer, softmax layer, and classification layer) are replaced with three new layers to adapt to the new dataset. Since the initial network learned a rich set of image features, fine-tuning a network with transfer learning is able to learn features specific to the new dataset. After that, train the pre-trained $\mathrm{CNNs}$ on the FishNet dataset to predict and assess the network accuracy.

The experiments were run on a desktop computer with Intel-i7 processor and 64 GB of RAM. During training, the hyper-parameters are adjusted to minimize training loss, which constitutes classification error. The initial learning rate was 0.0001 . A similar batch size, 100 , is employed and trained on 20 epochs to compare training time for each network. The network was trained with stochastic gradient descent with 0.9 momentums and the training was terminated after 4,080 iterations. CNN final output comprised a dimension, where $n$ represents the number of fish species. Through the fully connected layer, the network is able to transform the original image input to produce class scores for classification accuracy performance. Finally, a new test image is fed into the network to compare performance evaluations.

In this paper, we discuss fish image classification methods that utilize the softmax function. Softmax is a classifier that is able to produce class-probabilities (Springenberg et al., 2014). The softmax layer gives the output as the probability distribution over different labels. The softmax function is often used in multiple classification logistic regression models. The softmax function specifies a discrete problem probability distribution for $K$ classes, defined as (Agarap, 2018):

$$
\sum_{K=1}^{K} \rho k
$$

The equation denotes as the activation at the penultimate layer of a neural network, and as its weight parameters at the softmax layer, thus producing $\mathbf{0}$ as the input to the softmax layer.

$$
o=\sum_{i}^{n-1} \theta_{i} x_{i}
$$

Consequently, the equation will be:

$$
\rho k=\frac{\exp \left(o_{k}\right)}{\sum_{k=0}^{n-1} \exp \left(o_{-} k\right)}
$$

Hence, the predicted class would be $\hat{y}$,

$$
\hat{y}=\frac{\arg \arg \max }{i \in 1, \ldots, N} \rho i
$$

Confusion matrix is a table to describe classification accuracy performance. A confusion matrix is commonly known as a contingency table in which the matrix could be arbitrarily large (Santra \& Christy, 2012). Basic model performance measures, such as accuracy, error-rate, specificity, sensitivity, and precision, are derived from the confusion matrix. The basic measure classifiers that are most widely used are accuracy (ACC) and error rate (ERR) (Saito \& Rehmsmeier, 2015). The accuracy is defined as;

$$
\text { Accuracy }=\frac{(\text { True Positive }+ \text { True Negative })}{(\text { True Positive }+ \text { True Negative }+ \text { False Negative }+ \text { False Positive })}
$$




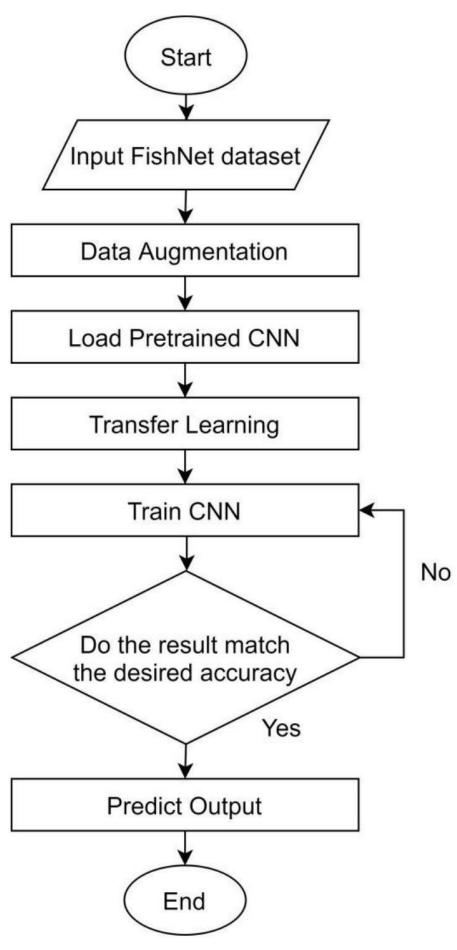

Figure 2: Flowchart of fish image classification study

\section{Result and Discussion}

This experiment was aimed to evaluate the performance of different pre-trained $\mathrm{CNN}$ using transfer learning when predicting the fish species. The performance of the models for fish image classification through overall classification accuracy, learning curves, classification accuracy from the validation dataset, top-five accuracy from test dataset and confusion matrix was analyzed. Table 5 shows the accuracy and training time for all pre-trained networks used in this study. It is observed that the best performance was obtained by AlexNet, followed by ResNet-50 and GoogLeNet, with an accuracy of $99.85 \%, 99.51 \%$, and $96.39 \%$ respectively. The classification accuracy is the percentage of correctly recognized fish species. Regarding the different pre-trained CNN, better performance was obtained with the AlexNet model, followed by ResNet-50 and GoogLeNet. This result shows that the performance of methods is related to the complexity of the network. The additional layers or more complex architecture will not ensure higher accuracy of prediction (Ayob et al., 2019).

Table 5 shows the training time of each pre-trained network obtained by AlexNet, GoogLeNet, and ResNet-50, with duration of 352 minutes 49 seconds, 662 minutes 59 seconds, and 1,743 minutes 27 seconds respectively. AlexNet requires the least training time to complete the learning methods due to the lowest depth of the network. It can be seen that the training time was tied to the total number of architecture layers of each pre-trained CNN; AlexNet had 8 layers, GoogLeNet had 22 layers and ResNet-50 had 50 layers. Fewer layer in the network contributes to better fish image classification performance.

The overall performance of models was analyzed by using a learning curve. The learning curve provides mathematical representations of the training plot that takes place as task classification. Figure 3 illustrates the learning curves for monitoring the performance of fish image classification tasks. Figure 3(a), Figure 3(b) and Figure 3(c) show the learning curve of training loss and validation loss over a number of iterations for AlexNet, GoogLeNet and ResNet-50 respectively. Figure 3(a) illustrates the plot of training loss decreasing to a point of stability. Meanwhile the plot of validation loss decreases to a point of stability and has

Table 5: Performance comparison of each pre-trained networks

\begin{tabular}{|c|c|c|}
\hline Pre-trained Networks & $\begin{array}{c}\text { Classification accuracy } \\
(\%)\end{array}$ & Training Time \\
\hline AlexNet & 99.85 & $352 \min 49 \mathrm{sec}$ \\
\hline GoogLeNet & 96.39 & $662 \mathrm{~min} 59 \mathrm{sec}$ \\
\hline ResNet-50 & 99.51 & $1743 \mathrm{~min} 27 \mathrm{sec}$ \\
\hline
\end{tabular}


a minimal gap with the training loss. Figure 3(b) shows both the plot of training loss and validation loss decreasing to a point of stability and has a small gap between the two lines. In Figure 3(c), below, it can be seen that the plot of training loss and validation loss remain stable and has the smallest gap among the pre- trained CNN. Regarding the learning curves, it can be seen that the performance of AlexNet, GoogLeNet and ResNet-50 are remarkably fitted, with sufficient information of training and validation dataset to learn the fish image classification task.

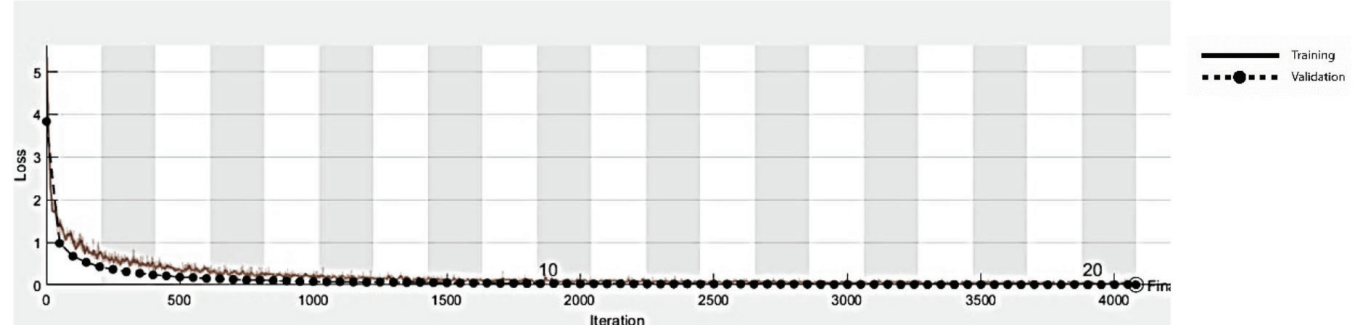

Figure 3(a): The training plot of loss over the number of iterations for AlexNet

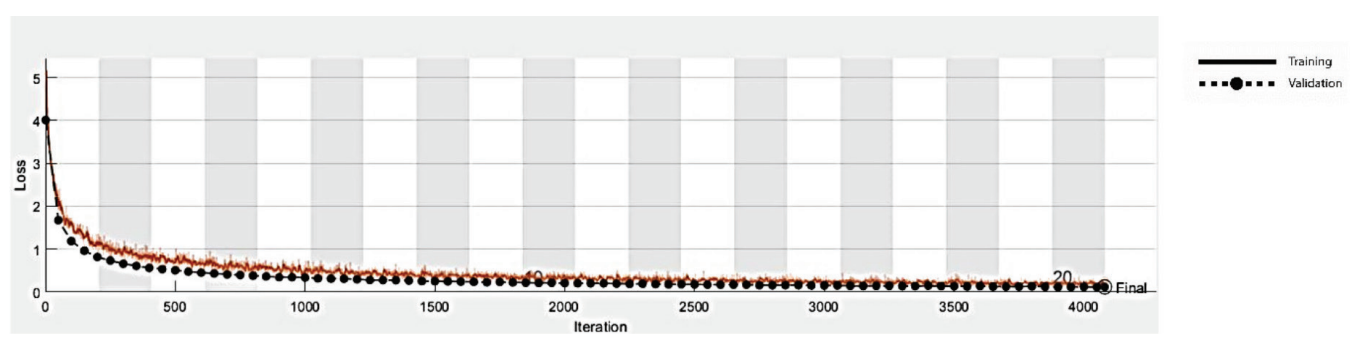

Figure 3(b): The training plot of loss over the number of iterations for GoogLeNet

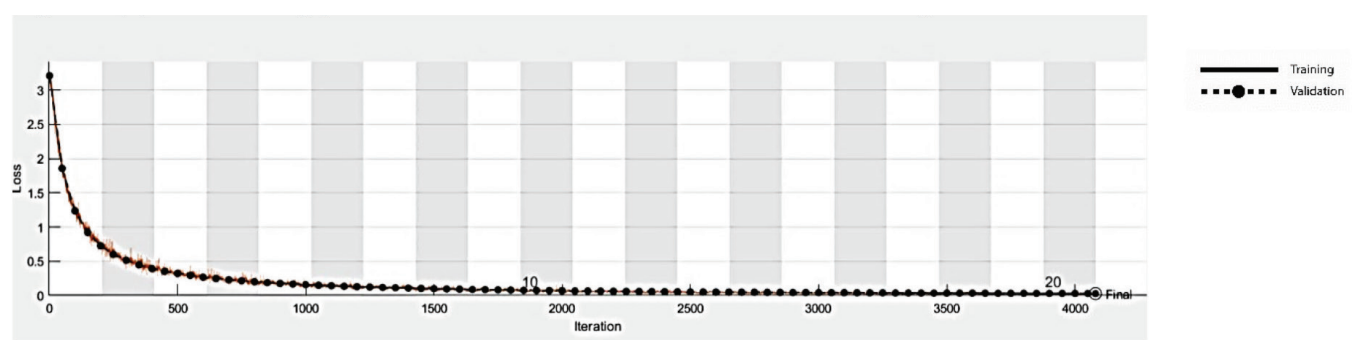

Figure 3(c): The training plot of loss over the number of iterations for ResNet-50

After that, deep learning models for each type of fish were evaluated using a validation image from each species. A validation set is used to monitor the model performance during the training process and fine tune hyper parameters. The accuracy results for each type of fish species are tabulated in Table 6. It can be seen that AlexNet has the best accuracy as it shows better classification in comparison to the other architecture, whereas the least accurate architecture is ResNet-50. The results show that
AlexNet has $100 \%$ correctly classified 11 fish species out of 18 species. Meanwhile, ResNet-50 has incorrectly classified 2 fish species that are Caranx ruber and Scomber scombrus which was misclassified as Rastrelliger kanagurta. This is the expected result, given the visually similar features of fish images in the dataset. Both Epinephelus fasciatus and Monodactylus argenteus have $100 \%$ accuracy on all the pretrained $\mathrm{CNN}$ as it has a significant color feature that differentiates them from other species. 
Table 6: Accuracy results for each type of fish on a validation dataset

\begin{tabular}{llll}
\hline & \multicolumn{1}{c}{ AlexNet (\%) } & \multicolumn{1}{c}{ GoogLeNet (\%) } & \multicolumn{1}{c}{ ResNet-50 (\%) } \\
\hline Atule mate & 91.70 & 73.00 & 62.20 \\
Caranx ruber & 83.60 & 74.00 & $(91.70)$ \\
Cetoscarus bicolor & 100.00 & 99.80 & 100.00 \\
Decapterus russelli & 97.40 & 91.00 & 93.80 \\
Epinephelus areolatus & 100.00 & 99.90 & 99.30 \\
Epinephelus fasciatus & 100.00 & 100.00 & 100.00 \\
Halichoeres argus & 99.90 & 88.70 & 98.20 \\
Hemiglyphidodon & & & \\
plagiometopon & 100.00 & 98.60 & 92.80 \\
Lutjanus inermis & 100.00 & 98.90 & 96.00 \\
Megalaspis cordyla & 99.70 & 53.90 & 99.90 \\
Monodactylus argenteus & 100.00 & 100.00 & 100.00 \\
Parastromateus niger & 100.00 & 99.20 & 99.90 \\
Pomacentrus alexanderae & 100.00 & 99.30 & 95.10 \\
Rastrelliger kanagurta & 99.90 & 93.00 & 99.90 \\
Salmo salar & 100.00 & 93.00 & 99.90 \\
Scomber Scombrus & 100.00 & 93.80 & $(63.60)$ \\
Scomberoides & & & 66.10 \\
commersonnianus & 63.30 & 50.50 & 95.90 \\
Scomberoides tala & 100.00 & 98.70 & \\
\hline
\end{tabular}

After that, performance of the pre-trained model with an image of test data (Figure 4) was evaluated. Figure 5 displays the top five predicted labels and their associated probabilities. The results suggest that ALexNet correctly classifies the image as Rastrelliger kanagurta with $69.9 \%$ probability and

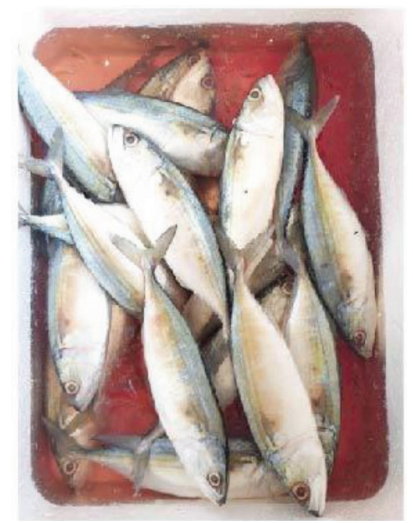

Figure 4: A test image of Rastrelliger kanagurta
ResNet-50 classifies the image as a Rastrelliger kanagurta with a $30.0 \%$ probability, which is misclassified as Atule mate. GoogLeNet classifies the image as a Rastrelliger kanagurta with the lowest probability (3.5\%). It can be seen that AlexNet model shows a high probability of correctly classifying fish species, while GoogLeNet and ResNet-50 were incorrect. This is expected as the test image was visually similar to the characteristic of Atule mate and has almost the same color and shape features.

The confusion matrix obtained from this experiment is shown in Figure 6, where along the $\mathrm{x}$-axis are listed the target class labels and along the $y$-axis are the output class predictions. Along the first diagonal section are the correct classifications, meanwhile, all the other entries show classification incorrectly. The bottom right cell of the confusion matrix shows the overall classification accuracy. In this confusion matrix, Figure 6(a), it can be 


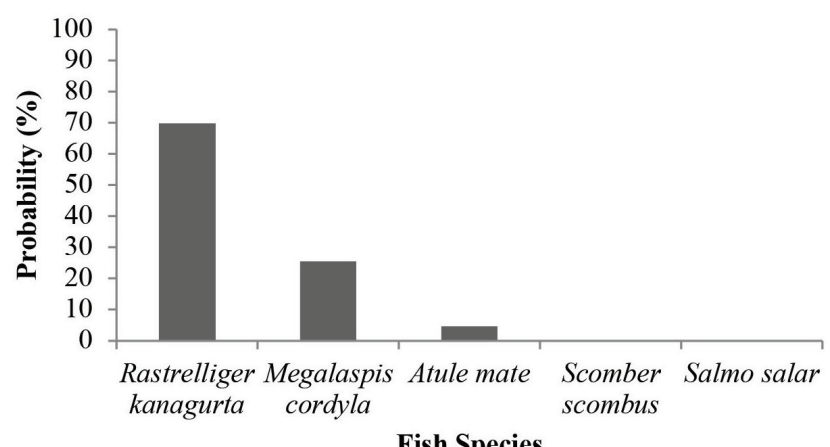

Figure 5(a): AlexNet correctly classified the image as a Rastrelliger kanagurta with $69.9 \%$ probability

\section{Top 5 Prediction (GoogLeNet)}

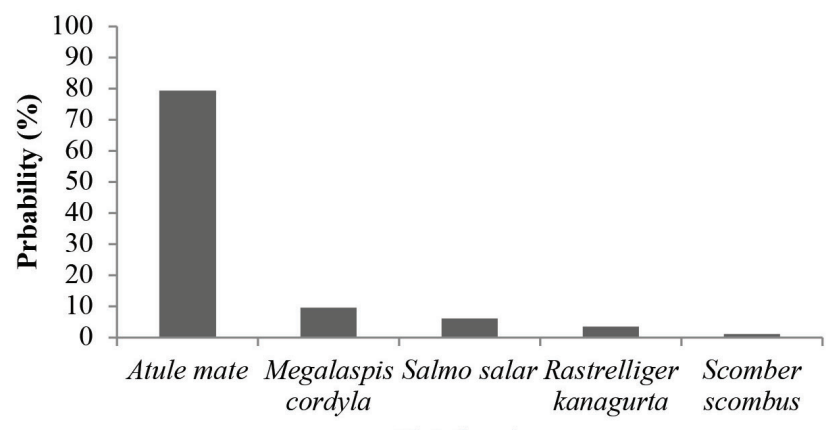

Fish Species

Figure 5(b): GoogLeNet classified the image as a Rastrelliger kanagurta with a 3.5\% probability and classified incorrectly as Atule mate

\section{Top 5 Prediction (ResNet-50)}

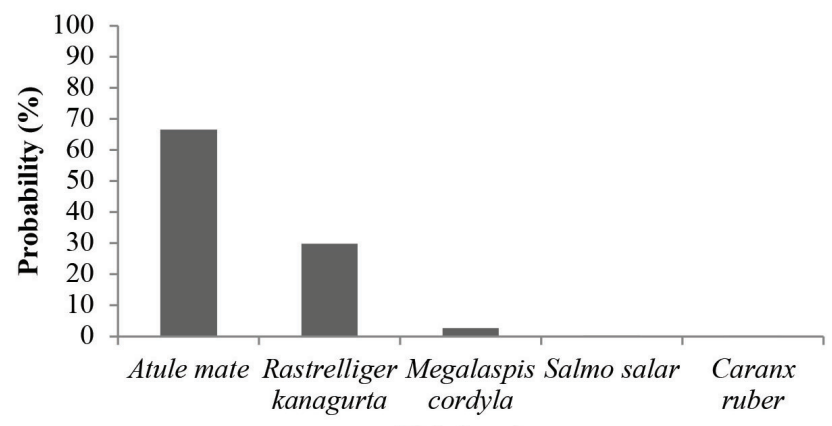

Fish Species

Figure 5(c): ResNet-50 classified the image as a Rastrelliger kanagurta with a 30.0\% probability and classified incorrectly as Atule mate 
seen that Decapterus russelli and Megalaspis cordyla have 3 examples wrongly predicted as Rastrelliger kanagurta. Figure 6(b) shows that Atule mate has 35 examples wrongly predicted as Rastrelliger kanagurta. Additionally, from the classification, Decapterus russelli has 29 examples wrongly predicted as Rastrelliger kanagurta. As illustrated in Figure 6(c), Atule mate has 6 examples wrongly predicted as Rastrelliger kanagurta. It can be observed that both GoogLeNet and ResNet-50 have incorrectly classified Atule mate as Rastrelliger kanagurta as shown in the top-five accuracy result.

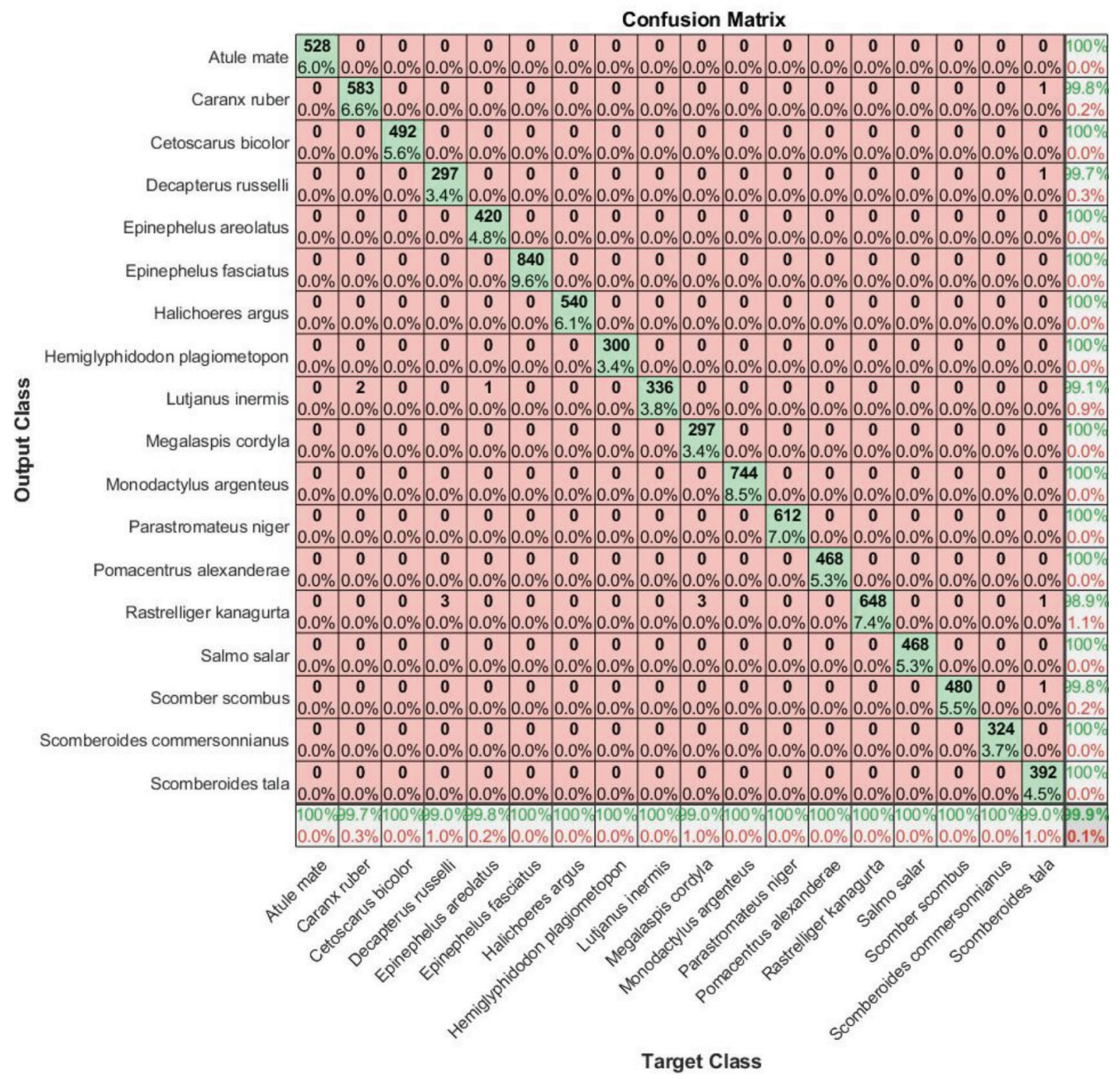

Figure 6(a): Confusion matrix for all classes and all attributes (AlexNet) 


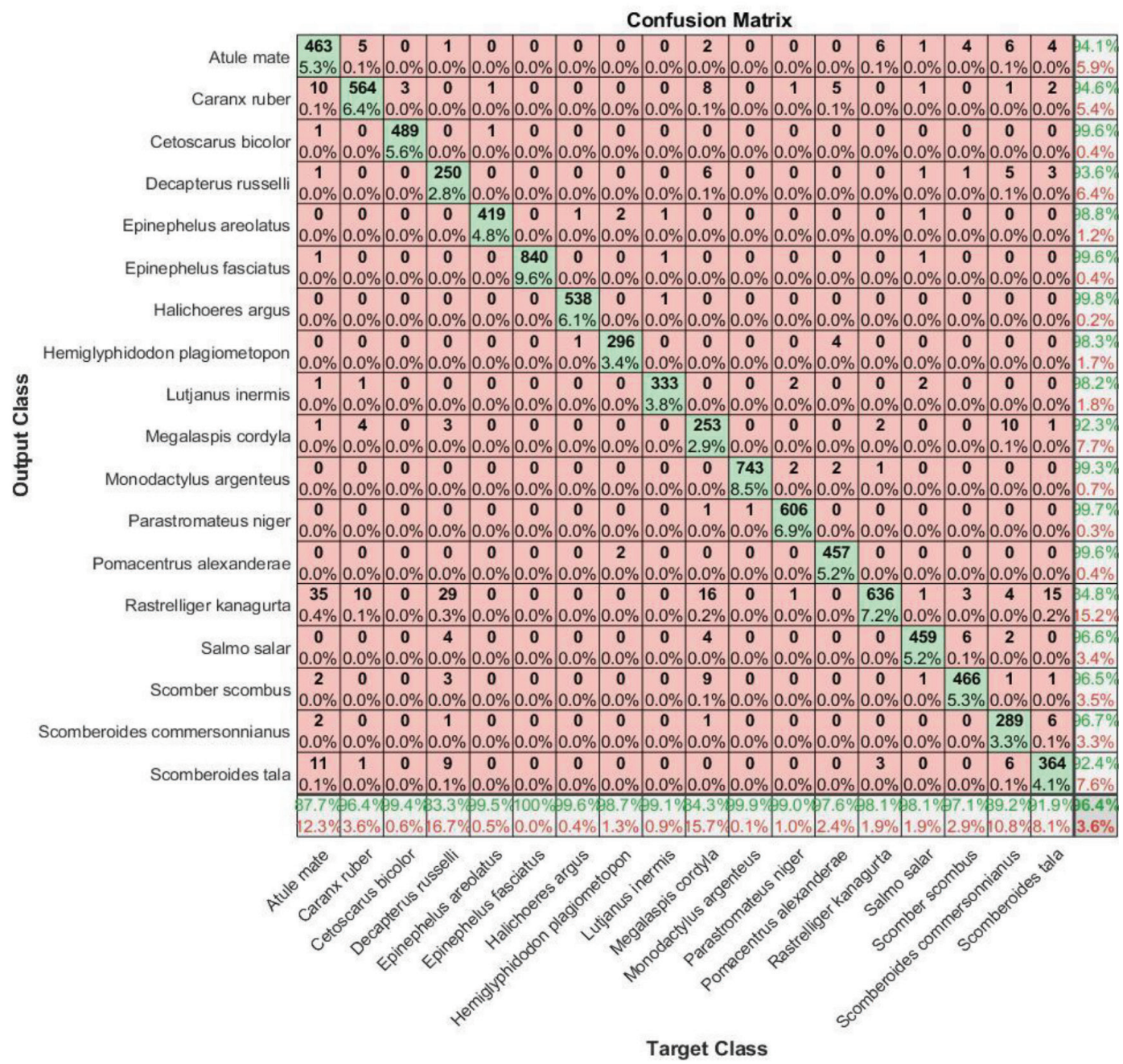

Figure 6(b): Confusion matrix for all classes and all attributes (GoogLeNet)

\section{Conclusion}

Presented is a model performance evaluation of pre-trained $\mathrm{CNN}$ architectures using transfer learning for fish image classification. The models' performance evaluation for fish image classification by using transfer learning provides the classification accuracy of more than $96 \%$ for 18 fish species. The training compared three different $\mathrm{CNN}$ models with depth ranging from 5 to 49 convolutional layers. The deepest architecture, ResNet-50, has the smallest gap in the plot of training loss and validation loss plot and requires more training time to achieve the maximum performance. Meanwhile the accuracy gain becomes higher in the test dataset evaluation with the least convolutional layers. AlexNet correctly classified the image as Rastrelliger kanagurta with $69.9 \%$ probability, ResNet-50 with a $30.0 \%$ probability, and GoogLeNet with a $3.5 \%$ probability. Both GoogLeNet and ResNet-50 classified the image incorrectly as Atule mate. It can be seen that, during validation and test dataset evaluation, the AlexNet model showed a high probability of correctly classifying fish species; while GoogLeNet and ResNet-50 output misclassified the result despite the high classification accuracy. This paper is an extensive study for each of 


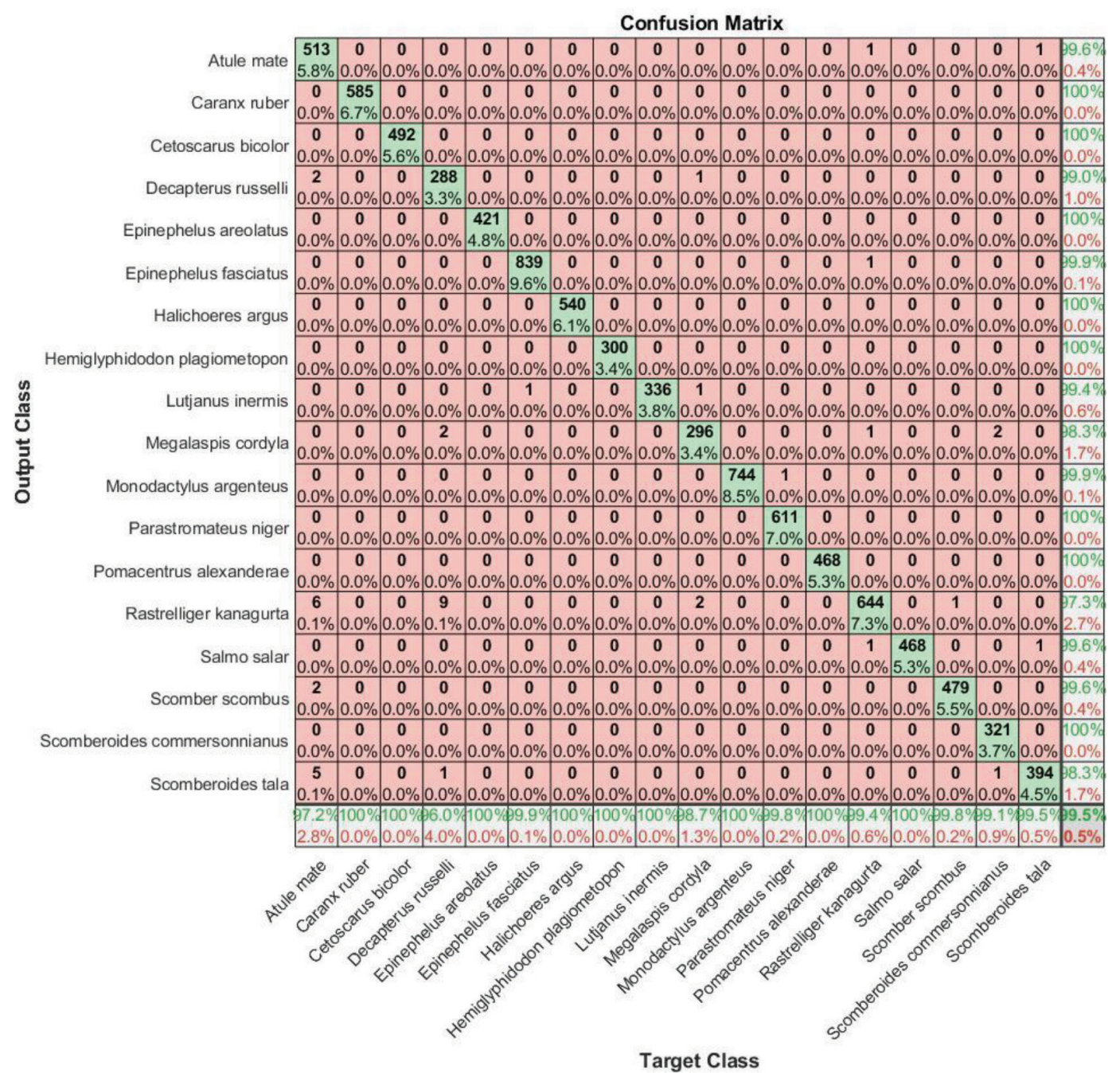

Figure 6(c): Confusion matrix for all classes and all attributes (ResNet-50)

the architecture which includes; classification accuracy, learning curve, validation test, topfive prediction and confusion matrix. An accurate fish identification tool is necessary for both experts and non-experts to prevent species misidentification. Transfer learning was able to reduce the training time and improve the performance of deep learning models. These models were able to quickly distinguish the different fish species phenotypes despite a limited quantity of labelled data.

\section{Acknowledgements}

Authors wished to thank the reviewers and editors for the constructive comments as well as the university for the research opportunity.

\section{References}

Agarap, A. F. (2018). Deep learning using rectified linear units (relu), arXiv preprint arXiv:1803.08375.

Alom, M. Z., Taha, T. M., Yakopcic, C., Westberg, S., Sidike, P., Nasrin, M. S., ... \& Asari, V. K. (2018). The history began 
from alexnet: A comprehensive survey on deep learning approaches. arXiv preprint arXiv:1803.01164.

Anantharajah, K., Ge, Z., McCool, C., Denman, S., Fookes, C., Corke, P., ... \& Sridharan, S. (2014). Local inter-session variability modelling for object classification. In IEEE Winter Conference on Applications of Computer Vision (pp. 309-316). IEEE.

Ayob, A. F., Khairuddin, K., Mustafah, Y. M., Salisa, A. R., \& Kadir, K. Analysis of Pruned Neural Networks (MobileNetV2-YOLO v2) for Underwater Object Detection. In Proceedings of the 11th National Technical Seminar on Unmanned System Technology 2019 (pp. 87-98). Springer, Singapore.

Choi, S. (2015). Fish Identification in Underwater Video with Deep Convolutional Neural Network: SNUMedinfo at LifeCLEF Fish task 2015. In CLEF (Working Notes).

Dangelo, M., \& Tennefoss, J. Plankton Species Identification via Convolutional Neural Networks.

Gómez-Ríos, A., Tabik, S., Luengo, J., Shihavuddin, A. S. M., Krawczyk, B., \& Herrera, F. (2019). Towards highly accurate coral texture images classification using deep convolutional neural networks and data augmentation. Expert Systems with Applications, 118, 315-328.

He, K., Zhang, X., Ren, S., \& Sun, J. (2016). Deep residual learning for image recognition. In Proceedings of the IEEE conference on computer vision and pattern recognition (pp. 770-778).

Hernández-Serna, A., \& Jiménez-Segura, L. F. (2014). Automatic identification of species with neural networks. PeerJ, 2, e563.

Huang, Z., Pan, Z., \& Lei, B. (2017). Transfer learning with deep convolutional neural network for SAR target classification with limited labeled data. Remote Sensing, 9(9), 907.
Kozłowski, M., Górecki, P., \& Szczypiński, P. M. (2019). Varietal classification of barley by convolutional neural networks. Biosystems Engineering, 184, 155-165.

Krähenbühl, P., Doersch, C., Donahue, J., \& Darrell, T. (2015). Data-dependent initializations of convolutional neural networks. arXiv preprint arXiv:1511.06856.

Kratzert, F., \& Mader, H. (2018). Fish species classification in underwater video monitoring using Convolutional Neural Networks.

Krizhevsky, A., Sutskever, I., \& Hinton, G. E. (2012). Imagenet classification with deep convolutional neural networks. In Advances in neural information processing systems (pp. 1097-1105).

Liawatimena, S., Heryadi, Y., Trisetyarso, A., Wibowo, A., Abbas, B. S., \& Barlian, E. (2018). A Fish Classification on Images using Transfer Learning and Matlab. In 2018 Indonesian Association for Pattern Recognition International Conference (INAPR) (pp. 108-112). IEEE.

Liu, Z. (2020). Soft-shell shrimp recognition based on an improved AlexNet for quality evaluations. Journal of Food Engineering, 266, 109698.

Man, M.,Abdullah, N., Rahim, M. S. M., \&Amin, I. M. (2016). Fish Length Measurement: The Results from Different Types of Digital Camera. Journal of Advanced Agricultural Technologies Vol, 3(1), 67-71.

Nadarajah, P. (1980). Species of Endogonaceae and mycorrhizal association of Elaeis guineensis and Theobroma cacao. Species of Endogonaceae and mycorrhizal association of Elaeis guineensis and Theobroma cacao., 232-237.

Polzounov, A., Terpugova, I., Skiparis, D., \& Mihai, A. (2016). Right whale recognition using convolutional neural networks. arXiv preprint arXiv:1604.05605.

Rathi, D., Jain, S., \& Indu, S. (2017). Underwater fish species classification 
using convolutional neural network and deep learning. In 2017 Ninth International Conference on Advances in Pattern Recognition (ICAPR) (pp. 1-6). IEEE.

Russakovsky, O., Deng, J., Su, H., Krause, J., Satheesh, S., Ma, S., ... \& Berg, A. C. (2015). Imagenet large scale visual recognition challenge. International journal of computer vision, 115(3), 211-252.

Saito, T., \& Rehmsmeier, M. (2015). The precision-recall plot is more informative than the ROC plot when evaluating binary classifiers on imbalanced datasets. PloS one, 10(3), $\mathrm{e} 0118432$.

Salman, A., Jalal, A., Shafait, F., Mian, A., Shortis, M., Seager, J., \& Harvey, E. (2016). Fish species classification in unconstrained underwater environments based on deep learning. Limnology and Oceanography: Methods, 14(9), 570-585.

Santra, A. K., \& Christy, C. J. (2012). Genetic algorithm and confusion matrix for document clustering. International Journal of Computer Science Issues (IJCSI), 9(1), 322.

Siddiqui, S. A., Salman, A., Malik, M. I., Shafait, F., Mian, A., Shortis, M. R., \& Harvey, E. S. (2018). Automatic fish species classification in underwater videos: exploiting pre-trained deep neural network models to compensate for limited labelled data. ICES Journal of Marine Science, 75(1), 374-389.

Springenberg, J. T., Dosovitskiy, A., Brox, T., \& Riedmiller, M. (2014). Striving for simplicity: The all convolutional net. arXiv preprint arXiv:1412.6806.

Steinbrener, J., Posch, K., \& Leitner, R.
(2019). Hyperspectral fruit and vegetable classification using convolutional neural networks. Computers and Electronics in Agriculture, 162, 364-372.

Szegedy, C., Liu, W., Jia, Y., Sermanet, P., Reed, S., Anguelov, D., ... \& Rabinovich, A. (2015). Going deeper with convolutions. In Proceedings of the IEEE conference on computer vision and pattern recognition (pp. 1-9).

Thenmozhi, K., \& Reddy, U. S. (2019). Crop pest classification based on deep convolutional neural network and transfer learning. Computers and Electronics in Agriculture, 164, 104906.

Tyagi, G., Patel, N., \& Sethi, I. (2018). A FineTuned Convolution Neural Network Based Approach For Phenotype Classification Of Zebrafish Embryo. Procedia Computer Science, 126, 1138-1144.

Verma, G. K., \& Gupta, P. (2018). Wild animal detection using deep convolutional neural network. In Proceedings of 2 nd international conference on computer vision \& image processing (pp. 327-338). Springer, Singapore.

Wang, S., Liu, H., Han, Y., Chen, J., Pan, Y., Cao, Y., ... \& Zheng, Y. (2018). Low-altitude remote sensing based on convolutional neural network for weed classification in ecological irrigation area. IFACPapersOnLine, 51(17), 298-303.

Xu, J. L., \& Sun, D. W. (2018). Computer vision detection of salmon muscle gaping using convolutional neural network features. Food analytical methods, 11(1), 34-47. 